showed that crossreactive anti-dsDNA/ NMDAR autoantibodies selectively damaged mouse hippocampal neurons. These antidsDNA/NMDAR-treated mice also performed more poorly than control mice (treated with anti-dsDNA/NMDAR ${ }^{-}$sera) in behavioral and cognitive tests that required flexible memory; however, both groups performed similarly on other memory-related tasks.

The authors also found that brain tissue from five deceased patients with neuropsychiatric SLE contained IgG that bound to both dsDNA and a pentapeptide derived from the NMDAR; these antibodies were not present in the brains of two patients with Parkinson's disease.

The authors conclude that crossreactive anti-DNA/NMDAR autoantibodies can cause neuronal damage in the brains of patients with SLE; however, this damage is contingent on the presence of a compromised BBB through which the antibodies can pass. The authors suggest that interventions to maintain the integrity of the BBB (e.g. control of blood pressure, smoking cessation, reduced stress) might prevent autoantibody-mediated damage to the central nervous system.

Original article Kowal C et al. (2006) Human lupus autoantibodies against NMDA receptors mediate cognitive impairment. Proc Natl Acad Sci USA 103: 19854-19859

\section{Internet-delivered activity programs might benefit patients with RA}

Patients with rheumatoid arthritis (RA) have low levels of physical activity despite extensive evidence that exercise has RA-specific benefits. Promising results for internet-delivered activity programs have been achieved in the general population, but no data had been collected on their use in patients with RA.

In a randomized, controlled trial that compared two internet-delivered activity programs, 160 physically inactive patients with RA received either an individualized $(n=82)$ or generalized $(n=78)$ intervention. In the individualized-intervention group, a tailor-made exercise program was accessible online, advice from physical therapists was sent weekly via email, and patient-therapist group meetings were held every 3 months. In the generalizedintervention group, general exercise information was accessible online, and patients were recommended to perform moderate-intensity physical activity for a 30 min period daily, 5 days a week. All patients were requested to complete a physical-activity schedule weekly. An activity monitor was worn continuously (except in water) for five consecutive days at baseline, 6 months and 12 months.

Patients in the individualized-intervention group reported a higher level of physical activity than patients in the generalized-intervention group (35\% versus $11 \%$ at 9 months), although activity monitoring showed no difference between the groups. There was a trend towards greater improvements in functional ability and quality of life in the individualized-intervention group than in the generalized-intervention group, but statistical significance was not achieved. The authors conclude that internet-based, individualized activity programs are a promising intervention to promote exercise in patients with RA.

Original article van den Berg MH et al. (2006) Using internet technology to deliver a home-based physical activity intervention for patients with rheumatoid arthritis: a randomized controlled trial. Arthritis Rheum 55: 935-945

\section{Rituximab affects both humoral and cellular immunity in patients with SLE}

B-cell depletion with rituximab is a promising therapy for patients with systemic lupus erythematosus (SLE) that has failed to respond to conventional disease-modifying antirheumatic drugs. Although B-cell depletion reduces autoantibody titers and improves disease symptoms for these patients, the effects of rituximab therapy on the whole immune system remain unclear. Vallerskog and colleagues, therefore, performed a longitudinal study to investigate changes in the immune system of patients with SLE in response to rituximab treatment.

Eleven women with treatment-refractory SLE received four once-weekly infusions of rituximab $375 \mathrm{mg} / \mathrm{m}^{2}$; cyclophosphamide $0.5 \mathrm{~g} / \mathrm{m}^{2}$ was included in the first and last infusions. Patients were followed up for 6-30 months. $\mathrm{B}$-cell and T-cell populations as well as antibody titers were measured at baseline, after B-cell depletion, during B-cell recovery, and after recovery. The median time to B-cell repopulation was 7.5 months (range 4-12 months); the fewer B cells the patient had initially, the longer it took for them to return. 